\title{
What Is Your Diagnosis? Prepubic mass in a mare.
}

\author{
Marco Tecilla', Matteo Gambini' ${ }^{1}$, Claudio Pigoli' ${ }^{1}$, Valeria Grieco${ }^{1}$, Mario Caniatti ${ }^{1}$ \\ ${ }^{1}$ Università degli Studi di Milano (UNIMI), Dipartimento di Medicina Veterinaria \\ (DIMEVET)
}

Corresponding author: Marco Tecilla, marco.tecilla@gmail.com

\section{CASE PRESENTATION}

A 30-year old Thoroughbred mare was presented to the referring veterinarian with a complaint of acute onset of anorexia, depression, weakness, and abdominal distention. Clinical signs were present for two weeks and progressed rapidly, but the mare had no history of previous disease. Rectal palpation revealed the presence of a round, immobile mass, associated with the cranial pelvic floor. Transrectal ultrasound characterized the mass as well-defined, heterogeneous, $20-\mathrm{cm}$ in diameter mass. Greater than 20 liters of pale-yellow fluid were removed via abdominal paracentesis. Differential diagnoses included a neoplastic or inflammatory process arising from either the urinary bladder, the genital apparatus, or the inguinal lymph nodes. Twenty milliliters of abdominal fluid were collected in both EDTA-coated and plain blood collection tubes and were submitted for cytological evaluation within two hours of sampling. Direct smear and cytocentrifuge preparations were prepared and manually-stained with May-Grünwald-Giemsa according to standard procedures (Merck KGaA, Frankfurt, Germany) (Fig. 1A, B).

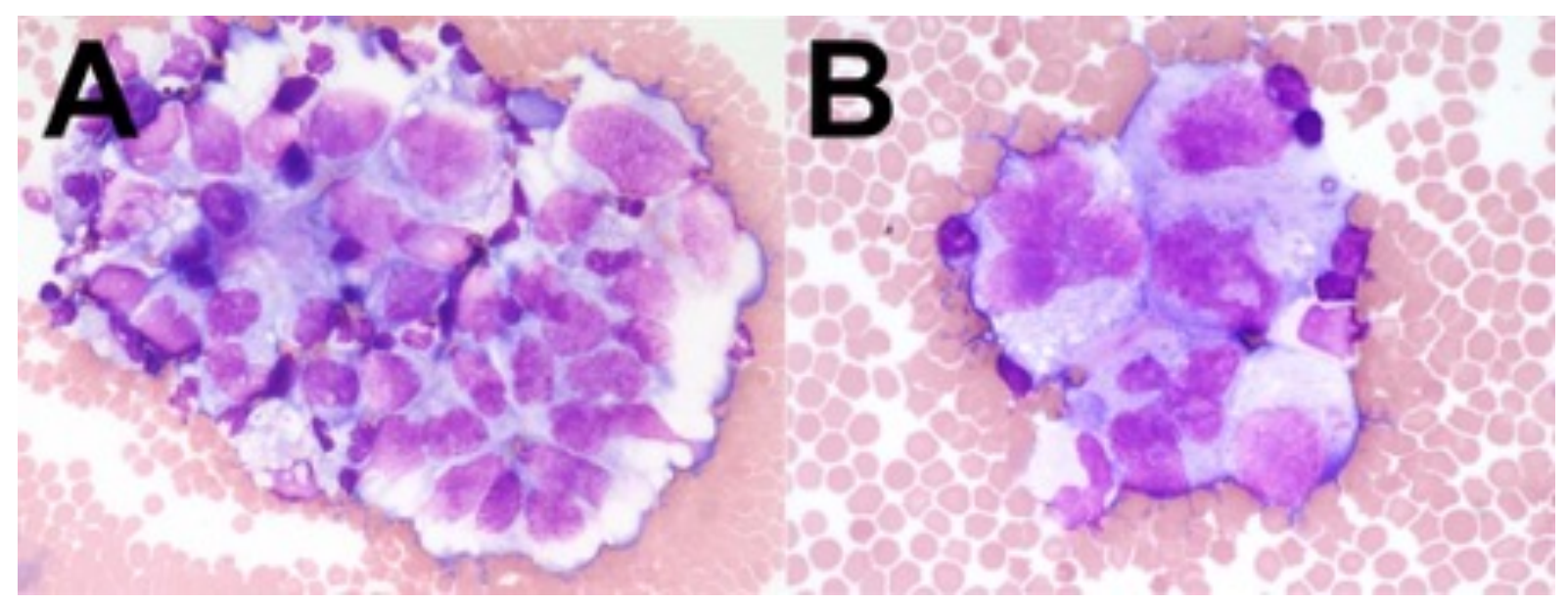

Figure 1: Abdominal fluid, mare. Cytospin ${ }^{\circledR}$, May-Grünwald-Giemsa (A) 40x objective. (B) 60x objective. 


\section{Cytologic interpretation:}

Poorly differentiated malignant tumor, of probable round-cell origin.

The cytological samples were hemodiluted and moderately cellular with a predominant population of large, round to atypical polygonal cells, noted individually, in clusters, or in rosette-like arrangements. Neoplastic cells exhibited moderate to marked anisocytosis and anisokaryosis, with variable nuclear-cytoplasmic ratio and variably distinct cell borders. The cytoplasm was scant to abundant, pale blue, with occasional, usually small, clear vacuoles. The nucleus was paracentral, round to irregular to convoluted, with finely granular, irregularly condensed chromatin, and multiple variably sized, light blue nucleoli. Multinucleate atypical cells were present in low numbers. Other cells included small mature lymphocytes, non-degenerate neutrophils, foamy macrophages, and reactive mesothelial cells. Due to the presence of atypical cells found in aggregates and rosette-like structures, which suggested an epithelial origin, immunocytochemistry for Pan-cytokeratin (clones AE1/AE3; Dako, Carpinteria, USA) was performed and was negative.

Based on the cytological diagnosis and clinical presentation, the owner declined additional testing or surgery. Abdominal fluid was drained daily to improve the mare's comfort. Because of clinical deterioration, the mare was euthanized and submitted for necropsy. At necropsy, the abdominal cavity was filled with more than fifteen liters of abdominal fluid. A $20-\mathrm{cm}$ in diameter mass was identified in association with the right ovary. The mass partially occupied the cranial pelvis and caudal prepubic/pubic regions. Multiple nodular lesions associated with the parietal peritoneum, liver, spleen, and intestine, compatible with carcinomatosis, were also detected. No other gross lesions were detected. On histopathology, the right ovary was completely replaced by a densely cellular neoplasm arranged in sheets, cords, rosettes and pseudorosettes, supported by moderate fibrovascular stroma (Fig. 2A, B). Neoplastic cells were round to polygonal, with indistinct cell borders, scant to moderately abundant vacuolated eosinophilic cytoplasm, and a paracentral, large, round to indented to clover leaf-shaped nucleus. One to three basophilic, 2-5 $\mu$ m, nucleoli were variably present. Anisokaryosis and anisocytosis were marked (Fig. 2C). Mitoses were 35 in 10 HPF (total area examined $2.37 \mathrm{~mm}^{2}$ ), with a range of 1-5 mitosis per field, and were frequently 
atypical. Numerous, multinucleated, neoplastic cells with up to 5 nuclei were randomly distributed. Multifocal random areas of necrosis and numerous, disseminated apoptotic neoplastic cells were also evident. Scattered aggregates of small mature lymphocytes, were frequently associated with apoptotic neoplastic cells. Peritoneal metastases were histologically identical to the primary mass. Lymphatic emboli were detected multifocally. Immunohistochemistry was performed on the ovarian neoplasm including Placental Alkaline Phosphatase (PLAP - polyclonal; Dako, Carpinteria, USA), Inhibin (clone R1; Serotec Co., UK), and Pan-Cytokeratin (clone AE1/AE3; Dako, Carpinteria, USA), and were were multifocally positive (Fig. 3), negative, and negative, respectively. The final diagnosis was ovarian dysgerminoma.

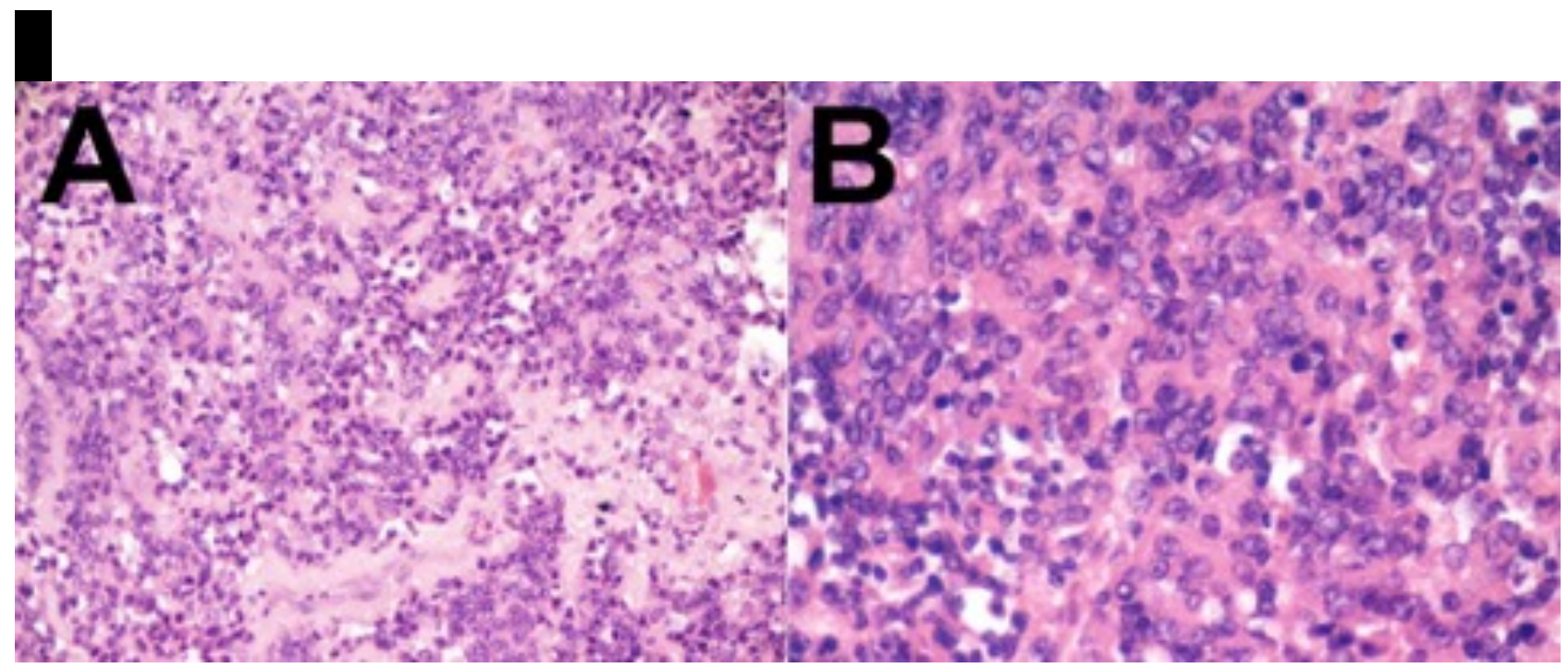

Figure 2: Ovarian dysgerminoma, mare. Sheets of neoplastic cells supported by a moderate fibrovascular stroma. Hematoxylin and Eosin (A) 20x objective. (B) 40x objective.

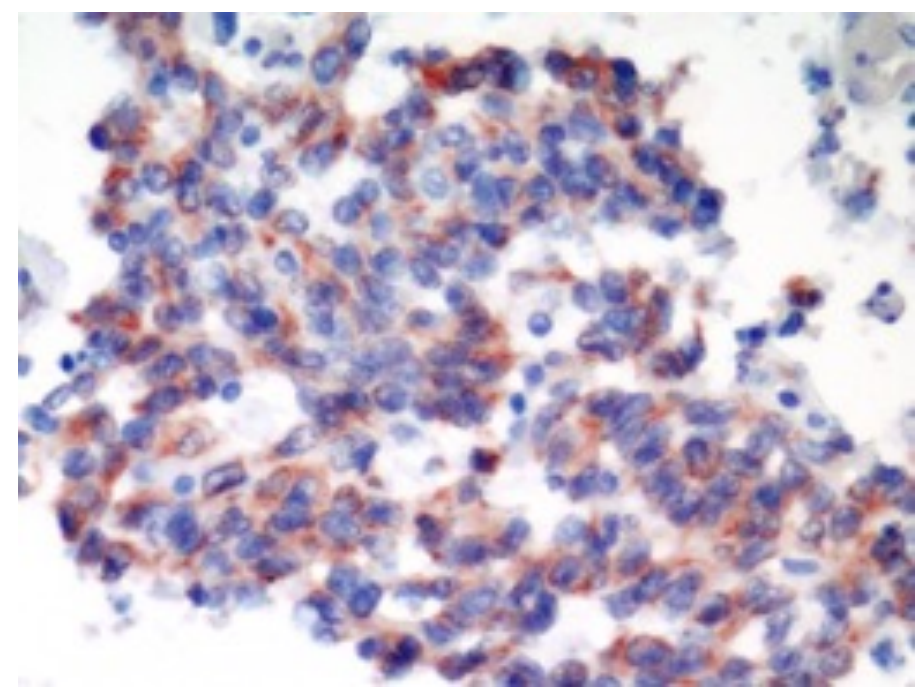


Figure 3: Immunohistochemical staining for anti-PLAP antibody, 40x objective. Multifocal, intense, membranous to cytoplasmic immune-positive reaction to PLAP is present.

\section{DISCUSSION}

Dysgerminomas are considered to be rare neoplasms in domestic animals, with only a few reports in horses..$^{3,4}$

However, dysgerminoma should be considered in the list of differentials for large, single, masses arising in the prepubic/pubic regions of mares. Adult or geriatric onset of marked abdominal effuision, in absence of systemic lymphadenopathy, is also suggestive of this diagnosis. ${ }^{4}$

Dysgerminomas are germ line tumors, and are the counterpart of seminomas of males. ${ }^{5}$ Germ line tumors are highly aggressive, metastasizing via both peritoneal seeding and lymphatics emboli. ${ }^{1}$ In the present case, lymphatic metastasis and carcinomatosis were likely responsible for the onset of the abdominal effusion. Cytologically, the presence of large atypical and pleomorphic cells, with loss of cohesivity, may lead to a diagnosis of a poorly differentiated round-cell tumor, particularly lymphoma as reported in dogs and cats. ${ }^{5}$ Although the cellular morphology seems compatible, one of the most consistant features of dysgerminoma is the high nuclear pleomorphism, which is less common in lymphoma, along with the abundant cytoplasm and the presence of scattered, small, mature lymphocytes. Additionally, dysgerminoma is one of the few neoplasm where histology is more helpful in excluding lymphoma, because of the presence of rosettes and pseudo-rosettes that are not a feature of lymphomas. The use of Anti-CD3 (T-cells) and Anti-CD20 or Anti-CD79 (Bcells) is not described in equine cytology but can be performed on histological sections to exclude definitively lymphoma. Multinucleated neoplastic cells or the presence of pink material in the cytoplasm, are inconstant cytological features, but are more readily detected on histology, because of the amount of tissue evaluated and absence of dilution. An additional differential diagnosis for dysgerminoma, based on the anatomical location and specific prevalence, is granulosa cell tumor. Although most granulosa cell tumors are characterized by the presence of Call-Exner bodies, both cytologically and histologically, a moderate level of cellular pleomorphism can be present, and this entity might be excluded by immunocytochemistry or immunohistochemistry. ${ }^{2}$ In cases where the difference between dysgerminoma and granulosa cell tumors cannot be excluded base on morphology, Anti-PLAP and Anti-Inhibin can be used to discriminate these entities. Anti-PLAP antibody recognizes a group of highly conserved membrane glycoproteins, normally expressed in the placenta, cervix and fallopian tubes. From a diagnostic point of view, PLAP is expressed by 
most male and female germline tumors including dysgerminomas and seminomas. PLAP reactivity can be membranous or cytoplasmic. In contrast, Inhibin is more specific for sex-chord tumors, such as Sertoli cell and granulosa cell tumors. Dysgerminoma is positively identified by PLAP positivity and inhibin negativity. Pan-cytokeratin can also be helpful, since most granulosa cell tumors are Pan-cytokeratin positive, but dysgerminomas are negative.

To the authors' knowledge, this is the first report describing cytological features of dysgerminoma in horses.

\section{Reference:}

1. Agnew D, MacLachlan N. Tumors of genital system. In: Meuten D, ed. Tumors in domestic animals. Ames: Willey Blackwell; 2016:698.

2. Ellenberger C, Bartmann CP, Hoppen HO, et al. Histomorphological and Immunohistochemical Characterization of Equine Granulosa Cell Tumours. J Comp Pathol. 2007;136(2-3):167-176.

3. Harland S, Smith C, Mogg T, Horadagoda N, Dart A. Surgical resection of a dysgerminoma in a mare. Aust Vet J. 2009;87(3):110-112.

4. Knottenbelt D, Snalune K, Patterson Kane J. Gonadal neoplasms. In: Knottenbelt D, Snalune K, Patterson Kane J, eds. Clinical Equine Oncology. St. Luis: Elsevier; 2015:400-401.

5. Raskin, RE Meyer D, editor. Reproductive system. In: Caniene and feline cytology. A color atlas and interpertation guide. Srt. Luis: Elsevier; 2016:325-326. 